\title{
A Characterization of Clustering States
}

\author{
Derek W. Robinson \\ Dept. de Physique, Université d'Aix-Marseille II, Marseille-Luminy, France
}

Received July 15, 1974

\begin{abstract}
A characterization of states, over quasi-local algebras, which satisfy a strong cluster property is derived. The discussion is applicable to classical systems and quantum systems with Bose or Fermi statistics.
\end{abstract}

\section{Introduction}

Several years ago Powers characterized primary states over UHF algebras as the states satisfying a certain singly uniform cluster property [1]. The importance of cluster properties in physical theories led several authors to generalize Powers results to other algebras encountered in field theory or statistical mechanics (see for example [2-6]). These generalizations took several different directions; the idea of far away observables, and analogy with Sinai's results on $K$-systems [7], is emphasized in [2,3]; the notion of relative commutants of observables is used in [4]; the singly uniform clustering property is equated with a doubly uniform clustering property in $[5,6]$. Most of these generalizations were, however, modeled on certain commutation properties which are typically encountered in classical mechanics or quantum mechanics with Bose statistics. The only attempt to characterize clustering states of Fermi systems occurs in [5] which considers only the even states of the CAR-algebra. Also the proof of the result concerning these states, Proposition 4.5 of [5], is incomplete ${ }^{1}$.

The purpose of this note is to correct this situation by providing a general discussion which applies to all standard systems encountered in statistical mechanics regardless of statistics.

\section{Quasi-local Systems}

In this section we discuss the basic structure of algebras which are generated by local subalgebras and possibly satisfy Fermi statistics.

Throughout $\mathscr{F}$ will denote an index set with an order relation $\geqq$. We always assume that $\mathscr{F}$ is directed with respect to this relation, i.e. if $\alpha, \beta \in \mathscr{F}$ then there exists a $\gamma \in \mathscr{F}$ such that $\gamma \geqq \alpha, \beta$. In the second half of this section we also assume the existence of an orthogonality relation $\perp$ between pairs of elements of $\mathscr{F}$ with the following properties

a) if $\alpha \leqq \beta$ and $\beta \perp \gamma$ then $\alpha \perp \gamma$,

b) if $\alpha \perp \beta$ and $\alpha \perp \gamma$ there exists a $\delta \in \mathscr{F}$ such that $\alpha \perp \delta$ and $\delta \geqq \beta, \gamma$.

\footnotetext{
$1 \mathfrak{A}\left(M_{a_{0}}\right)^{-}$and $\mathfrak{A}\left(M_{a}\right)^{\prime} \wedge \mathfrak{U}^{-}$do not generate $\mathfrak{U}_{a}^{-}$as is claimed in the proof given in [5].
} 
In the next section we further assume that each pair $\alpha, \beta \in \mathscr{F}$ has a unique least upper bound $\alpha \vee \beta \in \mathscr{F}$, i.e. $\alpha \vee \beta \in \mathscr{F}$ is the unique element such that every $\gamma \geqq \alpha, \beta$ satisfies $\gamma \geqq \alpha \vee \beta(\geqq \alpha, \beta)$.

Typically, in applications, $\mathscr{F}$ is the set of bounded open subsets of $\mathbb{R}^{v}$ or the set of finite subsets of $\mathbb{Z}^{v}$. The relation $\alpha \leqq \beta$ corresponds to inclusion of $\alpha$ in $\beta$, $\alpha \perp \beta$ corresponds to the disjointness of $\alpha$ and $\beta$, and $\alpha \vee \beta$ corresponds to the union of $\alpha$ and $\beta$.

Statistics will be introduced into our formalism by aid of an automorphism $\sigma$, of a $C^{*}$-algebra $\mathfrak{A}$, which satisfies $\sigma^{2}=1$, i.e.

$$
\sigma(\sigma(A))=A, \quad A \in \mathfrak{U} .
$$

Each element $A \in \mathfrak{U}$ has a unique decomposition into odd and even parts $A^{\mp}$ with respect to $\sigma$. This decomposition is defined by

$$
\begin{aligned}
A & =A^{+}+A^{-} \\
A^{ \pm} & =(A \pm \sigma(A)) / 2 .
\end{aligned}
$$

It follows that the even elements of $\mathfrak{A}$ form a $C^{*}$-subalgebra $\mathfrak{U}^{e}$ of $\mathfrak{A}$ and the odd elements a Banach space $\mathfrak{U}^{0}$. Now we introduce the class of algebras which will be studied in the sequel.

A $C^{*}$-algebra $\mathfrak{U}$ is defined to be quasi-local if it possesses a family $\left\{\mathfrak{U}_{\alpha}\right\}_{\alpha \in \mathscr{F}}$ of $C^{*}$-subalgebras. These subalgebras satisfy certain requirements such as

L.1 $\bigcup_{\alpha \in \mathscr{F}} \mathfrak{U}_{\alpha}$ is norm-dense in $\mathfrak{U}$.

L.2 If $\alpha \geqq \beta$ then $\mathfrak{U}_{\alpha} \supseteqq \mathfrak{U}_{\beta}$.

L.3 There exists an automorphism $\sigma$ of $\mathfrak{A}$ such that $\sigma^{2}=1$ and $\sigma\left(\mathfrak{U}_{\alpha}\right)=\mathfrak{U}_{\alpha}$ for all $\alpha \in \mathscr{F}$.

L. $4^{2}$ If $\alpha \perp \beta$ then the following commutation relations are valid

$$
\begin{gathered}
{\left[\mathfrak{U}_{\alpha}^{e}, \mathfrak{Y}_{\beta}^{e}\right]=0, \quad\left[\mathfrak{H}_{\alpha}^{e}, \mathfrak{A}_{\beta}^{0}\right]=0} \\
\left\{\mathfrak{U}_{\alpha}^{0}, \mathfrak{U}_{\beta}^{0}\right\}=0 .
\end{gathered}
$$

We remark that in classical mechanics, or quantum mechanics with Bose statistics, $\sigma$ is the identity automorphism, thus $\mathfrak{U}_{\alpha}=\mathfrak{U}_{\alpha}^{e}$; for Fermi statistics $\sigma$ is non-trivial and corresponds to a change of sign of the Fermi creation and annihilation operators.

Following [2] we now associate with each representation $\pi$ of the quasi-local algebra $\mathfrak{U}$ the algebra of observables at infinity $\mathfrak{B}^{\pi}$ by the definition

$$
\mathfrak{B}^{\pi}=\bigcap_{\alpha \in \mathscr{F}}\left(\bigcup_{\beta \perp \alpha} \pi\left(\mathfrak{U}_{\beta}\right)\right)^{-} .
$$

This algebra has the following properties

Proposition $1 .^{3}$ Let $\omega$ be a state over a quasi-local algebra $\mathfrak{A}$ which satisfies L.1-L.4. Then the algebra at infinity $\mathfrak{B}^{\pi_{\omega}}$ is contained in the centre $3_{\pi_{\omega}}$ of $\pi_{\omega}^{\prime \prime}$ and

${ }^{2}$ We use the usual notation $[A, B]=A B-B A,\{A, B\}=A B+B A$.

${ }^{3} \pi_{\omega}$ always refers to the Hilbert space representation of $\mathfrak{P}$ on $\mathscr{H}_{\omega}$ canonically associated with $\omega$. 
in particular

$$
\begin{aligned}
\mathfrak{B}^{\pi_{\omega}} & =\bigcap_{\alpha \in \mathscr{F}}\left(\bigcup_{\beta \perp \alpha} \pi_{\omega}\left(\mathfrak{U}_{\beta}^{e}\right)\right)^{\prime \prime} \\
& \cong 3_{\omega} \cap \pi_{\omega}\left(\mathfrak{A}^{e}\right)^{\prime \prime} .
\end{aligned}
$$

The following conditions are equivalent

1. $\mathfrak{B}^{\pi_{\omega}}$ consists of multiples of the identity

2. Given $\varepsilon>0$ and $A \in \mathfrak{A}$ there exists an $\alpha \in \mathscr{F}$ such that

for all

$$
|\omega(A B)-\omega(A) \omega(B)|<\varepsilon\|B\|
$$

$$
B \in \bigcup_{\beta \perp \alpha} \mathfrak{A}_{\beta}
$$

3. Given $\varepsilon>0$ and $A \in \mathfrak{A}$ there exists an $\alpha \in \mathscr{F}$ such that

for all

$$
|\omega(A B)-\omega(A) \omega(B)|<\varepsilon\|B\|
$$

$$
B \in \bigcup_{\beta \perp \alpha} \mathfrak{P}_{\beta}^{e}
$$

Proof. Once we establish that

$$
\mathfrak{B}^{\pi_{\omega}} \subseteq 3 \pi_{\omega}
$$

then the equivalence of the Conditions 1 and 2, or 1 and 3, follow from Theorem 5.1 of [3]. Thus we concentrate on the characterization of $\mathfrak{B}^{\pi_{\omega}}$.

If $\alpha \in \mathscr{F}$ and $B \in \mathfrak{B}^{\pi_{\omega}}$ are given, one can choose a sequence $B_{n} \in \pi_{\omega}\left(\mathfrak{U}_{\alpha_{n}}\right)$ such that the $\alpha_{n}$ are mutually orthogonal, $\alpha_{n} \perp \alpha$, and $B_{n}$ converges strongly to $B$. (First one selects $\alpha_{1}$ and $B_{1}$ such that $\alpha_{1} \perp \alpha$ and $\left\|\left(B_{1}-B\right) \psi\right\|<1$, then $\alpha_{2}$ and $B_{2}$ such that $\alpha_{2} \perp \alpha_{1}, \alpha_{2} \perp \alpha$ and $\left\|\left(B_{2}-B\right) \psi\right\|<1 / 2$ etc. .) It then follows from the uniform boundedness principle that $\left\|\sigma\left(B_{n}\right)\right\|=\left\|B_{n}\right\| \leqq b$ with $b$ independent of $n$. Thus, possibly passing to a subsequence, we can assume the $\sigma\left(B_{n}\right)$ converge weakly. In particular, the odd and even parts $B_{n}^{\mp}$ of $B_{n}$ converge weakly. As the $\alpha_{n}$ are mutually orthogonal condition L.4 implies

$$
\left(B_{n}^{-}\right)^{*} B_{m}^{-}+B_{m}^{-}\left(B_{n}^{-}\right)^{*}=0
$$

for $n \neq m$ and this allows the estimation (see $[8,9]$ )

$$
\begin{aligned}
\left\|\frac{1}{N} \sum_{n=1}^{N} B_{n}^{-}\right\|^{2} & \leqq \frac{1}{N^{2}}\left\|\sum_{n, m=1}^{N}\left(B_{n}^{-}\right)^{*} B_{m}^{-}+B_{m}^{-}\left(B_{n}^{-}\right)^{*}\right\| \\
& \leqq \frac{2}{N} b .
\end{aligned}
$$

Thus the $B_{n}^{-}$converge weakly, their Cesaro means converge uniformly to zero, and hence $B_{n}^{-}$must converge to zero. Therefore, $B_{n}^{+}$converges weakly to $B$ and we have established that

$$
B \in\left(\bigcup_{\beta \perp \alpha} \pi_{\omega}\left(\mathfrak{U}_{\beta}^{e}\right)\right)^{\prime \prime} \cong \pi_{\omega}\left(\mathfrak{U}_{\alpha}\right)^{\prime} \cap \pi_{\omega}\left(\mathfrak{U}^{e}\right)^{\prime \prime} .
$$


But this is valid for all $\alpha \in \mathscr{F}$ and hence we conclude that

$$
\begin{aligned}
\mathfrak{B}^{\pi_{\omega}} & \cong \bigcap_{\alpha \in \mathscr{F}}\left(\bigcup_{\beta \perp \alpha} \pi_{\omega}\left(\mathfrak{A}_{\beta}^{e}\right)\right)^{\prime \prime} \\
& \cong \pi_{\omega}(\mathfrak{A})^{\prime} \cap \pi_{\omega}\left(\mathfrak{A}^{e}\right)^{\prime \prime} .
\end{aligned}
$$

The first inclusion is valid in the opposite sense by definition and hence must be an equality. This completes the characterization of $\mathfrak{B}^{\pi_{\omega}}$.

\section{Locally Normal States}

In this section we place further restrictions on the quasi-local structure of the algebra $\mathfrak{A}$ and states $\omega$ which we consider. These conditions are arranged so that any of the usual algebras associated with the canonical commutation, or anticommutation relations, are covered.

We need the following condition on the local subalgebras $\mathfrak{A}_{\alpha}$ of the quasilocal algebra $\mathfrak{A}$.

L.5 Each $\mathfrak{H}_{\alpha}$ is isomorphic to an irreducible subalgebra $\pi\left(\mathfrak{H}_{\alpha}\right)$ of the algebra $\mathfrak{B}\left(\mathscr{H}_{\alpha}\right)$ of all bounded operators on some Hilbert space $\mathscr{H}_{\alpha}$. There exist $R_{\alpha}=R_{\alpha}^{*} \in \mathfrak{B}\left(\mathscr{H}_{\alpha}\right)$ such that

$$
R_{\alpha}{ }^{2}=\mathbb{1}_{\mathscr{H}_{\alpha}}, \quad R_{\alpha} \pi(A) R_{\alpha}=\pi(\sigma(A))
$$

for all $A \in \mathfrak{A}_{\alpha}$. Further, on $\mathscr{H}_{\alpha \vee \beta}$ the $\pi\left(\mathfrak{H}_{\alpha}\right)^{-}$and $\pi\left(\mathfrak{H}_{\beta}\right)^{-}$are type I factors embedded in $\pi\left(\mathfrak{U}_{\alpha \vee \beta}\right)^{-}$with the property that

$$
\left(\pi\left(\mathfrak{H}_{\alpha}\right) \cup \pi\left(\mathfrak{A}_{\beta}\right)\right)^{-}=\pi\left(\mathfrak{H}_{\alpha \vee \beta}\right)^{-} .
$$

We have used a slightly over-simplified notation in this condition. What is meant is that the representation of $\mathfrak{A}_{\alpha}$, considered as a subalgebra of $\mathfrak{A}_{\alpha \vee \beta}$, on $\mathscr{H}_{\alpha \vee \beta}$ is a sum of copies of the representation of $\mathfrak{A}_{\alpha}$ on $\mathscr{H}_{\alpha}$.

A state $\omega$ over a quasi-local algebra $\mathfrak{A}$ which satisfies conditions L.1-L.5 is now defined to be locally normal if for each $\alpha \in \mathscr{F}$ one has

$$
\omega\left(\mathfrak{A}_{\alpha}\right)=\operatorname{Tr}_{\mathscr{H}_{\alpha}}\left(\varrho_{\alpha} \pi\left(\mathfrak{A}_{\alpha}\right)\right)
$$

where $\varrho_{\alpha}$ is a positive trace-class operator on $\mathscr{H}_{\alpha}$ with trace-norm unity.

Further the state is defined as continuous for the automorphism $\sigma$ if $\sigma$ is weak operator continuous in the representation $\pi_{\omega}$ generated by $\omega$. This will of course be the case if $\sigma$ is covariantly implemented, i.e. if there exists a unitary $U$ such that

$$
\pi_{\omega}(\sigma(A))=\bigcup \pi_{\omega}(A) U^{-1}
$$

for all $A \in \mathfrak{A}$, in particular if $\omega$ is even, i.e. invariant under $\sigma$.

Our immediate aim is to prove the following

Proposition 2. If $\omega$ is a locally normal state (over a quasi-local algebra $\mathfrak{A}$ satisfying conditions L.1-L.5), continuous for the automorphism $\sigma$, then the algebra 
of observables at infinity $\mathfrak{B}^{\pi_{\omega}}$ satisfies the following

$$
\mathfrak{B}^{\pi_{\omega}}=3_{\pi_{\omega}} \cap \pi_{\omega}\left(\mathfrak{Q}^{e}\right)^{\prime \prime}
$$

Lemma 1. Let $\omega$ be locally normal and $\alpha \perp \beta$. There is an $\hat{R}_{\alpha} \in \pi_{\omega}\left(\mathfrak{U}_{\alpha}\right)^{\prime \prime}$ such that $\hat{R}_{\alpha}^{2}=1$ and

Further one has

$$
\hat{R}_{\alpha} \pi_{\omega}(A) \hat{R}_{\alpha}=\pi_{\omega}(\sigma(A)), \quad A \in \mathfrak{U}_{\alpha} .
$$

$$
\pi_{\omega}\left(\mathfrak{U}_{\alpha}\right)^{\prime} \cap \pi_{\omega}\left(\mathfrak{U}_{\alpha \vee \beta}\right)^{\prime \prime}=\left(\hat{R}_{\alpha} \pi_{\omega}\left(\mathfrak{U}_{\beta}^{0}\right)+\pi_{\omega}\left(\mathfrak{A}_{\beta}^{e}\right)\right)^{\prime \prime} .
$$

Proof. As $\omega$ is normal in restriction to $\mathfrak{A}_{\alpha}$ the representation space $\mathscr{H}_{\omega}$ has a factorization

$$
\mathscr{H}_{\omega}=\mathscr{H}_{\alpha} \otimes \mathscr{H}^{\prime}
$$

and the representation $\pi_{\omega}$ restricted to $\mathfrak{A}_{\alpha}$ is then of the form

$$
\pi_{\omega}\left(\mathfrak{U}_{\alpha}\right)=\pi\left(\mathfrak{U}_{\alpha}\right) \otimes \mathbb{1}_{\mathscr{H}^{\prime}} .
$$

The $\hat{R}_{\alpha}$ is then defined by

$$
\hat{R}_{\alpha}=R_{\alpha} \otimes \mathbb{1}_{\mathscr{H}^{\prime}}
$$

and the first properties follow from L.5. To establish the second property we remark that $\pi_{\omega}\left(\mathfrak{U}_{\alpha \vee \beta}\right)$ is a sum of copies of $\pi\left(\mathfrak{U}_{\alpha \vee \beta}\right)$ and it suffices to prove the property for the latter representation. But a simple calculation using L. 4 demonstates that

$$
\left(R_{\alpha} \pi\left(\mathfrak{U}_{\beta}^{0}\right)+\pi\left(\mathfrak{U}_{\beta}^{e}\right)\right)^{\prime \prime} \subseteq \pi\left(\mathfrak{U}_{\alpha}\right)^{\prime} \cap \pi\left(\mathfrak{U}_{\alpha \vee \beta}\right)^{\prime \prime}
$$

Now $\pi\left(\mathfrak{U}_{\alpha}\right)^{\prime \prime}$ is embedded as a type I factor in $\pi\left(\mathfrak{U}_{\alpha \vee \beta}\right)^{\prime \prime}$. We have

$$
\begin{aligned}
\mathscr{H}_{\alpha \vee \beta} & =\mathscr{H}_{\alpha} \otimes \mathscr{H}^{\prime \prime} \\
\pi\left(\mathfrak{U}_{\alpha \vee \beta}\right) & =\pi\left(\mathfrak{U}_{\alpha}\right) \otimes \mathbb{1}_{\mathscr{H}^{\prime \prime}}
\end{aligned}
$$

and, as $\pi\left(\mathfrak{U}_{\alpha}\right)$ is irreducible, the left hand set occurring in $(*)$ must be of the form

$$
\mathbb{1}_{\mathscr{H}_{\alpha}} \otimes \mathscr{B}
$$

where $\mathscr{B}$ is a weakly closed subalgebra of $\mathfrak{B}\left(\mathscr{H}^{\prime \prime}\right)$. To deduce equality in (*) it suffices to prove that $\mathscr{B}$ is irreducible on $\mathscr{H}^{\prime \prime}$ or, equivalently, that

$$
\pi\left(\mathfrak{U}_{\alpha}\right) \cup\left(R_{\alpha} \pi\left(\mathfrak{U}_{\beta}^{0}\right)+\pi\left(\mathfrak{U}_{\beta}^{e}\right)\right)^{\prime \prime}
$$

is irreducible on $\mathscr{H}_{\alpha \vee \beta}$. But this latter set contains $\pi\left(\mathfrak{U}_{\alpha}\right) \cup \pi\left(\mathfrak{A}_{\beta}\right)$ and the result follows from Condition L.5.

Finally Proposition 2 is established by the second statement of the following.

Lemma 2. If $\omega$ is a locally normal state then

$$
3_{\pi_{\omega}}=\bigcap_{\alpha \in \mathscr{F}}\left(\bigcup_{\beta \perp \alpha} \pi_{\omega}\left(\mathfrak{H}_{\alpha}\right)^{\prime} \cap \pi_{\omega}\left(\mathfrak{\mathcal { U }}_{\alpha \vee \beta}\right)^{\prime \prime}\right)^{\prime \prime}
$$


and if, further, $\omega$ is continuous for the automorphism $\sigma$ then

$$
3 \pi_{\omega} \cap \pi_{\omega}\left(\mathfrak{U}^{e}\right)^{\prime \prime}=\bigcap_{\alpha \in \mathscr{F}}\left(\bigcup_{\beta \perp \alpha} \pi_{\omega}\left(\mathfrak{U}_{\beta}^{e}\right)\right)^{\prime \prime} .
$$

Proof. The first statement is proved in [4]. To deduce the second we first use Lemma 1 to identify

$$
3_{\pi_{\omega}}=\bigcap_{\alpha \in \mathscr{F}}\left(\bigcup_{\beta \perp \alpha}\left(\hat{R}_{\alpha} \pi_{\omega}\left(\mathfrak{H}_{\beta}^{0}\right)+\pi_{\omega}\left(\mathfrak{A}_{\beta}^{e}\right)\right)^{\prime \prime}\right)^{\prime \prime} .
$$

Secondly the continuity implies that the even part of the weak closure of a set of operators in the representation is the closure of the even part. Thirdly one has that $\hat{R}_{\alpha}$ is even because $\sigma$ applied to $\pi_{\omega}\left(\mathfrak{U}_{\alpha}\right)^{\prime \prime}$ is covariantly implemented by $\hat{R}_{\alpha}$ and

$$
\sigma\left(\hat{R}_{\alpha}\right)=\hat{R}_{\alpha}\left(\hat{R}_{\alpha}\right) \hat{R}_{\alpha}=\hat{R}_{\alpha} .
$$

The desired result follows immediately.

Proposition 2 follows directly from Proposition 1 and the second statement of Lemma 2.

Combination of Propositions 1 and 2 provides criteria for clustering of locally normal states. Stronger results can, however, be obtained by full exploitation of local normality as we show in the next section.

\section{Doubly Uniform Clustering}

The following result strengthens the statements, on clustering, obtained from Propositions 1 and 2.

Proposition 3. Let $\omega$ be a locally normal state (over a quasi-local algebra satisfying Conditions L.1-L.5) and continuous for the automorphism $\sigma$, then the following conditions are equivalent.

1. $\jmath_{\pi_{\omega}} \cap \pi_{\omega}\left(\mathfrak{U}^{e}\right)^{\prime \prime}$ consists of multiples of the identity.

2. Given $\varepsilon>0$ and $A \in \mathfrak{U}$ there is a $\beta \in \mathscr{F}$ such that

for all $B \in \bigcup_{\gamma \perp \beta} \mathfrak{A}_{\gamma}$

$$
|\omega(A B)-\omega(A) \omega(B)|<\varepsilon\|B\|
$$

3. Given $\varepsilon>0$ and $\alpha \in \mathscr{F}$ there is a $\beta \in \mathscr{F}$ such that

$$
|\omega(A B)-\omega(A) \omega(B)|<\varepsilon\|A\|\|B\|
$$

for all $A \in \mathfrak{U}_{\alpha}$ and all $B \in \bigcup_{\gamma \perp \beta} \mathfrak{A}_{\gamma}$

4. Given $\varepsilon>0$ and $A \in \mathfrak{A}$ there is $a \beta \in \mathscr{F}$ such that

for all $B \in \bigcup_{\gamma \perp \beta} \mathfrak{U}_{\gamma}^{e}$

$$
|\omega(A B)-\omega(A) \omega(B)|<\varepsilon\|B\|
$$

5. Given $\varepsilon>0$ and $\alpha \in \mathscr{F}$ there is $a \beta \in \mathscr{F}$ such that

$$
\left|\sum_{i=1}^{N} \omega\left(A_{i} B_{i}\right)-\omega\left(A_{i}\right) \omega\left(B_{i}\right)\right|<\varepsilon\left\|\sum_{i=1}^{N} A_{i} B_{i}\right\|
$$

for all $A_{i} \in \mathfrak{U}_{\alpha}, B_{i} \in \bigcup_{\gamma \perp \beta} \mathfrak{U}_{\gamma}^{e}$, and all $N$. 
The equivalence of Conditions 1, 2 and 4 is established by Propositions 1 and 2 . The remaining conditions both contain extra uniformity. A doubly uniform clustering property of the type considered in Condition 3 was first derived in [6] by remarking that the local normality allows the inner algebra $\mathfrak{U}_{\alpha}$ to be strongly approximated by a finite-dimensional algebra. This also allows the deduction of Condition $5^{4}$. This latter form of clustering was re-expressed, in [5], in terms of tensor product states and derived by an independent method which apparently does not allow the derivation of Condition 3. Thus we will base our proof of the proposition on the methods of [6] and use the tensor product structure suggested in [5] to make one of the auxiliary estimates.

For the proof it is convenient to introduce an auxiliary quasi-local $C^{*}$-algebra $\mathfrak{B}$ on $\mathscr{H}_{\omega}$. The algebra $\mathfrak{B}$ is defined in terms of local subalgebras $\left\{\mathfrak{B}_{\alpha}\right\}_{\alpha \in \mathscr{F}}$ with the choice

$$
\mathfrak{B}_{\alpha}=\pi_{\omega}\left(\mathfrak{U}_{\alpha}\right)^{\prime \prime}
$$

The state $\omega$ has an extension, also noted by $\omega$, to $\mathfrak{B}$ given by

$$
\omega\left(\mathfrak{B}_{\alpha}\right)=\operatorname{Tr}_{\mathscr{H}_{\alpha}}\left(\varrho_{\alpha} \mathfrak{B}_{\alpha}\right), \quad \alpha \in \mathscr{F}
$$

where $\varrho_{\alpha}$ are the density matrices determined by the original state. Note that $\mathfrak{U} \subset \mathfrak{B}$ and on $\mathscr{H}_{\omega}$ one has

$$
\mathfrak{B}^{\prime}=\pi_{\omega}(\mathfrak{U})^{\prime}, \quad \mathfrak{B}^{\prime \prime}=\pi_{\omega}(\mathfrak{U})^{\prime \prime}, \quad \text { etc. }
$$

In particular, the centre and the even part of the centre, of $\mathfrak{U}$ and $\mathfrak{B}$ represented on $\mathscr{H}_{\omega}$ are identical. Now we can consider the proposition re-expressed in terms of $\mathfrak{B}$ and $\omega$. It is then easily seen that if this latter form of the proposition can be established then the proposition, as stated, is also established. For this one uses the information already contained in Propositions 1 and 2, the containment $\mathfrak{A} \subset \mathfrak{B}$, and the equalities of the centres. Thus we will consider only the proposition concerning $\mathfrak{B}$, and prove that $2 \Rightarrow 3$ and $4 \Rightarrow 5$. The reverse implications are obvious and these equivalences suffice to complete the proof of the proposition. We need the following.

Lemma 3. Let $\varrho$ be a density matrix ${ }^{5}$ on a Hilbert space $\mathscr{H}$ and $R$ an operator on $\mathscr{H}$ with the properties $R=R^{*}, R^{2}=1$.

Given $\varepsilon>0$ there exists a finite-dimensional projector $E$ on $\mathscr{H}$ such that

and

$$
R E=E R
$$

$$
\operatorname{Tr}_{\mathscr{H}}(E \varrho E)>1-\varepsilon \text {. }
$$

Proof. Let $\varrho^{+}$be the even part of $\varrho$, with respect to the reflection induced by $R$, i.e.

$$
\varrho^{+}=(\varrho+R \varrho R) / 2 .
$$

It is evident that $\varrho^{+}$is positive and of trace-class.

\footnotetext{
4 This point was clarified by a discussion with J. Slawny in 1972 .

5 A positive, trace-class operator with trace-norm unity.
} 
As $\varrho^{+}$is even, i.e. commutes with $R$, its spectral projectors will also be even. Clearly there exists a finite dimensional spectral projector $E$ of $\varrho^{+}$such that

$$
\operatorname{Tr}_{\mathscr{H}}\left(E \varrho^{+} E\right)>1-\varepsilon .
$$

Next note that if $A$ is odd, i.e. $R A R=-A$, and of trace-class

$$
\operatorname{Tr}_{\mathscr{H}}(A)=-\operatorname{Tr}_{\mathscr{H}}(R A R)=-\operatorname{Tr}_{\mathscr{H}}(A)=0 \text {. }
$$

But $E\left(\varrho-\varrho^{+}\right) E$ is odd and of trace-class. Therefore

$$
\operatorname{Tr}_{\mathscr{H}}(E \varrho E)=\operatorname{Tr}_{\mathscr{H}}\left(E \varrho^{+} E\right)>1-\varepsilon .
$$

To deduce the implication $2 \Rightarrow 3$ we first let $E \in \pi\left(\mathfrak{U}_{\alpha}\right)^{\prime \prime}$ denote a finite dimensional even projector, i.e. $R_{\alpha} E R_{\alpha}=E$. (At the risk of a slight confusion $E$ will also denote the image of this projector in $\mathfrak{B}_{\alpha}$.) Given $\varepsilon>0$ we can, by Lemma 3 and local normality, choose $E$ such that

$$
\sqrt{\omega(1-E)}<\varepsilon / 12 \text {. }
$$

(The dimension $n$ of $E$ will then depend on $\varepsilon$.)

Next consider the decomposition

$$
\begin{aligned}
& \omega(A B)-\omega(A) \omega(B)= \omega(E A B E)-\omega(E A E) \omega(B) \\
&+\omega((1-E) A B E)-\omega((1-E) A E) \omega(B) \\
&+\omega(E A B(1-E))-\omega(E A(1-E)) \omega(B) \\
&+\omega((1-E) A B(1-E))-\omega((1-E) A(1-E)) \omega(B)
\end{aligned}
$$

where $A, B \in \mathfrak{B}$. One has

$$
\begin{aligned}
|\omega((1-E) A B E)| & \leqq \sqrt{\omega(1-E)}\|A\|\|B\| \\
|\omega((1-E) A E) \omega(B)| & \leqq \sqrt{\omega(1-E)}\|A\|\|B\| \quad \text { etc. }
\end{aligned}
$$

and hence

$$
|\omega(A B)-\omega(A) \omega(B)| \leqq|\omega(E A B E)-\omega(E A E) \omega(B)|+(\varepsilon / 2)\|A\|\|B\| .
$$

But if $A \in \mathfrak{B}_{\alpha}$ and $B \in \mathfrak{B}_{\beta}$ with $\beta \perp \alpha$ one can use the evenness of $E$ to deduce that

$$
\omega(E A B E)-\omega(E A E) \omega(B)=\omega\left(A_{E} B\right)-\omega\left(A_{E}\right) \omega(B)
$$

where $A_{E}=E A E$. Finally let $\left\{\psi_{k}\right\}_{k=1, \ldots, n}$ be an orthonormal basis for the range of $E$. One has

$$
A_{E}=\sum_{k, l=1}^{n} a_{k l} E_{k l}, \quad a_{k l}=\left(\psi_{k}, A \psi_{l}\right)
$$

where $E_{k l}$ denotes the matrix units associated with $\left\{\psi_{k}\right\}$. Therefore

$$
\left|\omega\left(A_{E} B\right)-\omega\left(A_{E}\right) \omega(B)\right| \leqq\|A\| n^{2} \sup _{k, l \leqq n}\left|\omega\left(E_{k l} B\right)-\omega\left(E_{k l}\right) \omega(B)\right| .
$$

But Condition 2 implies that $\beta \in \mathscr{F}$ can be chosen large enough that

$$
\left|\omega\left(E_{k l} B\right)-\omega\left(E_{k l}\right) \omega(B)\right|<\frac{\varepsilon}{2 n^{2}}\|B\|
$$

for all $B \in \bigcup_{\gamma \perp \beta} \mathfrak{B}_{\beta}$. Combining the above estimates yields Condition 3 . 
The proof that $4 \Rightarrow 5$ begins in a similar manner by the choice of $E$ and the decomposition for $\omega(A B)-\omega(A) \omega(B)$. One also has the estimates

$$
\left|\sum_{i=1}^{N} \omega\left((1-E) A_{i} B_{i} E\right)\right| \leqq \sqrt{\omega(1-E)}\left\|\sum_{i=1}^{N} A_{i} B_{i}\right\|
$$

but one needs the following

Lemma 4. If $\omega$ is locally normal, $C_{i} \in \mathfrak{A}_{\alpha}, B_{i} \in \mathfrak{A}_{\beta}^{e}$ with $\beta \perp \alpha$ then

$$
\left|\sum_{i=1}^{N} \omega\left((1-E) C_{i}\right) \omega\left(B_{i}\right)\right| \leqq \sqrt{\omega(1-E)}\left\|\sum_{i=1}^{N} C_{i} B_{i}\right\| .
$$

Proof. Consider the states $\omega_{\alpha}$ and $\omega_{\beta}$, over $\mathfrak{A}_{\alpha}$ and $\mathfrak{A}_{\beta}$, given by $\omega_{\alpha}=\left.\omega\right|_{\mathfrak{A}_{\alpha}}$ and

$$
\begin{aligned}
& \omega_{\beta}(B)=\omega(B) \quad \text { if } \quad B \in \mathfrak{A}_{\beta}^{e} \\
& =0 \text { if } B \in \mathfrak{A}_{\beta}^{0} .
\end{aligned}
$$

Let $\left(\mathscr{H}_{\alpha}, \pi_{\alpha}, \Omega_{\alpha}\right),\left(\mathscr{H}_{\beta}, \pi_{\beta}, \Omega_{\beta}\right)$ be the associated triplets and define the tensor product representation of $\mathfrak{A}_{\alpha \vee \beta}$, where $\alpha \perp \beta$, by

$$
\begin{aligned}
\mathscr{H} & =\mathscr{H}_{\alpha} \otimes \mathscr{H}_{\beta} & & \\
\pi(A) & =\pi_{\alpha}(A) \otimes \mathbb{1}, & & A \in \mathfrak{U}_{\alpha}^{e} \\
& =\pi_{\alpha}(A) \otimes R_{\beta}, & & A \in \mathfrak{H}_{\alpha}^{0} \\
\pi(B) & =\mathbb{1} \otimes \pi(B), & & B \in \mathfrak{U}_{\beta} .
\end{aligned}
$$

A straightforward calculation, using

then yields

$$
R_{\beta} \Omega_{\beta}=\Omega_{\beta}
$$

where

$$
(\Omega, \pi(A) \pi(B) \Omega)=\omega_{\alpha}(A) \omega_{\beta}(B)
$$

$$
\Omega=\Omega_{\alpha} \otimes \Omega_{\beta} .
$$

Thus, if $E, C_{i} \in \mathfrak{A}_{\alpha}$ and $B_{i} \in \mathfrak{A}_{\beta}^{e}$ one has

$$
\sum_{i=1}^{N} \omega\left((1-E) C_{i}\right) \omega\left(B_{i}\right)=\sum_{i=1}^{N}\left(\pi(1-E) \Omega, \pi\left(C_{i} B_{i}\right) \Omega\right)
$$

and the desired result follows from the Schwartz inequality applied to the right hand-side.

The remainder of the proof that $4 \Rightarrow 5$ is similar to that for $2 \Rightarrow 3$. Note that in terms of the matrix units

where

$$
\sum_{i=1}^{N} E A_{i} E B_{i}=\sum_{k, l=1}^{n} E_{k l} B_{k l},
$$

and hence

$$
B_{k l}=\sum_{i=1}^{N}\left(\psi_{k}, A_{i} \psi_{l}\right) B_{i} \in \mathfrak{B}_{\beta}
$$

$$
\left\|B_{k l}\right\| \leqq\left\|\sum_{i=1}^{N} A_{i} B_{i}\right\| .
$$


If $\omega$ is an even state, i.e. $\omega(A)=0$ for all $A \in \mathfrak{A}^{0}$ or, alternatively, $\omega(\sigma(A))=\omega(A)$ for all $A \in \mathfrak{U}$, then the results of Proposition 3 can be strenghened. One has

Proposition 4. Let $\omega$ be an even locally normal state (over a quasi-local algebra $\mathfrak{A}$ satisfying Conditions L.1-L.5) then the following conditions are equivalent

1. $3 \pi_{\omega} \cap \pi_{\omega}\left(\mathfrak{A}^{e}\right)^{\prime \prime}$ consists of multiples of the identity.

2. Given $\varepsilon>0$ and $\alpha \in \mathscr{F}$ there is a $\beta \in \mathscr{F}$ such that

$$
\left|\sum_{i=1}^{N} \omega\left(A_{i} B_{i}\right)-\omega\left(A_{i}\right) \omega\left(B_{i}\right)\right|<\varepsilon\left\|\sum_{i=1}^{N} A_{i} B_{i}\right\|
$$

for all $A_{i} \in \mathfrak{U}_{\alpha}, B_{i} \in \bigcup_{\gamma \perp \beta} \mathfrak{O}_{\gamma}$ and all $N$.

The proof that $2 \Rightarrow 1$ is implicit in Propositions 1 and 2. To prove the converse we proceed as in the proof of $4 \Rightarrow 5$ in Proposition 3 but note that the estimate of Lemma 4 is valid for all $B_{i} \in \mathfrak{A}_{\beta}$ if $\omega$ is even.

To conclude we remark that the statement of Proposition 4 differs from the result claimed in Proposition 4.5 of [5]. This latter result states that the above clustering property is equivalent to $z_{\omega}$ being multiples of the identity. The two results are not incompatible but, for them to be simultaneously true, it would be necessary that the centre of every locally normal even state is even. This appears to be unlikely. There certainly exist even quasi-free states whose centre is not even [10].

Acknowledgements. It is a pleasure to thank John Roberts for pointing out an error in an earlier version of this work.

\section{References}

1. Powers, R.: Ann. Math. 86, 138 (1967)

2. Lanford, O.E., Ruelle, D.: Commun. math. Phys. 13, 194 (1969). Naudts, J.: Ann. Soc. Sci. Brux. 84, III, 257 (1970)

3. Ruelle, D.: J. Funct. Ann. 6, 116 (1970)

4. Haag, R., Kadison, R. V., Kastler, D.: Commun. math. Phys. 16, 81 (1970)

5. Haag, R., Kadison, R. V., Kastler, D.: Commun. math. Phys. 22, 1 (1973)

6. Robinson, W.: Commun. math. Phys. 19, 219 (1970)

7. Sinai, Ya. G. : Amer. Math. Soc. Transl. (2) 31, 62 (1963)

8. Powers, R.: Thesis, Princeton Univ. (1967)

9. Lanford, O. E., D. W. Robinson: J. Math. Phys. 9, 1120 (1968)

10. Manuceau, J., Verbeure, A.: Commun. math. Phys. 18, 319 (1970) 